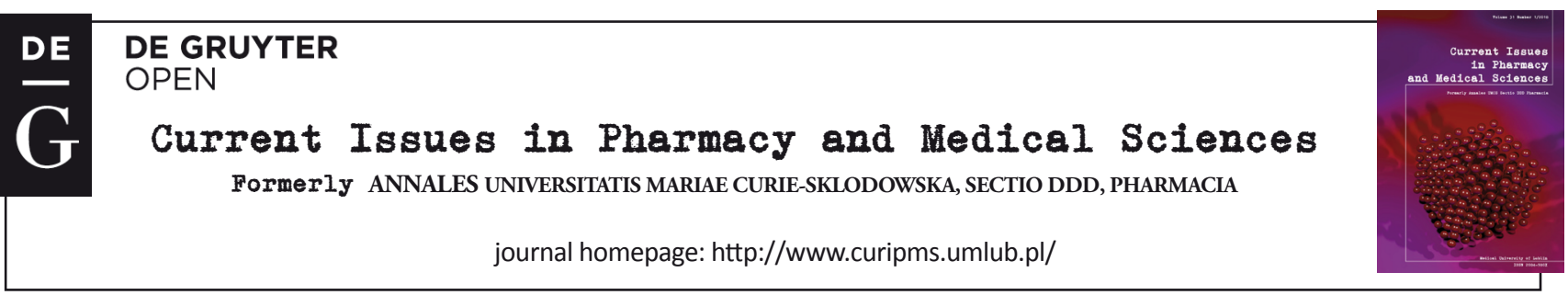

\title{
Apoptosis of rats' cardiomyocytes after chronic energy drinks consumption
}

\author{
Miroslaw Aleksander Slawinski, Ewelina Wawryk-Gawda*, \\ Michal Konrad Zarobkiewicz, Pawel Halczuk, Barbara Jodlowska-Jedrych
}

${ }^{1}$ Chair and Department of Histology and Embryology with Experimental Cytology Unit Medical University of Lublin, Radziwiłłowska 11, 20-080 Lublin, Poland

\section{ARTICLE INFO}

Received 13 September 2017 Accepted 18 December 2017

\section{Keywords:}

energy drink,

apoptosis,

cardiomyocytes,

p53 protein.

\begin{abstract}
Energy drinks (ED) are beverages containing caffeine, taurine, vitamins, herbal extracts, and sugar or sweeteners. They are marketed as capable of improving stamina, athletic performance and concentration, moreover, as serving as a source of energy. Still, there are very few papers describing the impact of ED on cell biology - including cell apoptosis within tissues. Therefore, in our study, we assessed the symptoms of rat cardiomyocytes apoptosis after 8 weeks consumption of ED.

For the research, we used male Wistar rats divided into 2 groups (experimental and control). The experimental animals received ED at a dose average of $0.190 \mathrm{ml}$ per $\mathrm{g}$ of body weight per day for a period of 8 weeks. The animals of the control group received just water and food without limitation. After 8 weeks, the rats were decapitated; hearts and other organs were collected. After embedding in paraffin blocks, $5 \mu \mathrm{m}$ thick tissue slides were prepared and stained according to standard hematoxylin and eosine (H\&E) staining protocol. Additional slides were stained by immunohistochemistry with antibodies directed against either caspaze- 3 or $\mathrm{p} 53$ protein.

Our results showed that the expression of caspase 3 and p53 protein varied depending on the group of rats. The expression of caspase 3 observed in cardiomyocytes was much more intense in the experimental group compared to the control group. Furthermore, the immunoprecipitation of $\mathrm{p} 53$ protein was observed more frequently in the cardiomyocytes nuclei of the experimental group than in the control group.

Obtained results suggest that chronic use of ED induces intracellular disorders and apoptosis in consumer cardiomyocytes.
\end{abstract}

\section{INTRODUCTION}

Energy drinks (ED) are defined as a beverages containing significant amounts of glucose, caffeine and taurine the manufacturers, however, also provide ED with herbs, vitamins and amino acids [1]. Due to the combination of these ingredients, ED are marketed as improving concentration and stamina, as well as reducing fatigue and increasing exercise tolerance. Therefore, they are often consumed by athletes, professional drivers and students [1-3]. Unfortunately, ED are frequently being drunk by children and adolescents [4]. Studies that have been carried out so far have focused on the clinical effects of the consumption of ED. The results indicate that ED consumption elevates blood pressure and heart rate, as well as increasing the heart load.

\footnotetext{
* Corresponding author

e-mail: ewelina.wawryk@wp.pl
}

Moreover, ED consumption has been linked with increased risk of traumatic brain injuries among adolescents [5-7]. The trend of combining ED with alcohol has created new perspectives for research and adoption of new clinical hypotheses [8]. The analysis of emergency department cases involving ED consumption revealed increased risk for complications among patients with pre-existing cardiovascular disease [9], and the possibility of ED being the cause of sudden death $[10,11]$. In our study, we analysed the histological changes in the cardiomyocytes and tried to link them with clinically observed symptoms.

Apoptosis is a programmed cell death, triggered to either physiologically end cell life or when some irreparable defects in DNA occurred. Caspases are enzymes belonging to the group of cysteine proteases which play an important role in that process. Initiator caspases are activated either by 
the intracellular or extracellular pathway. Effector caspases are responsible for the execution of apoptosis; caspase 3 belongs to this group. Caspase activity rise during apoptosis, and at certain point, they are blocked by endogenous caspase inhibitors [12]. Caspases bring about protein hydrolysis and resulting apoptosis-related programmed cell disintegration $[13,14]$. The increase of tissue caspase 3 concentration, therefore, is a sign of increase in apoptotic activity. The p53 protein is one of the major regulators of intracellular processes, including apoptosis. Its main function is triggering the repair process in situations of damage due to stressors acting on the cell. If the damages are irreparable, the p53 induces apoptotic cell death [15]. The half-life of the active form of this protein is short. Once the DNA damage is repaired, the p53 becomes deactivated. Therefore, the presence of active p53 within the nucleus should be considered as a sign of an ongoing pathological process which will result in either complete damage repair, or of programmed cell death $[16,17]$.

Thus, in our study, apart from standard H\&E, immunohistochemical stainings targeting p53 and caspase 3 were performed. Those stainings enabled an analysis of intracellular repair and apoptosis intensity within the cardiomyocytes of ED-consuming Wistar rats.

\section{MATERIALS AND METHODS}

\section{Experiment}

The experiment involved 10 Wistar rats (the mean weight: $155.4 \mathrm{~g}$ ) and lasted 8 weeks. During this period, all the observations were written in the study's diary. Before the experimental phase began, the animals were given a week to accommodate to the new surroundings. The rats were divided into two groups: the control and the experimental. The control group received just water for the following 8 weeks, while the rats of the experimental group received only energy drinks ad libitum (they had no access to water). Both water and ED were administered in special plastic drinkers hung on the cages. The drinkers were scaled so amount of consumed fluid could be measured. Herein, the obtained result was divided by the number of rats in the cage. The mean daily consumption was further reassured by repeated $24 \mathrm{~h}$-long stays in metabolic cages. The animals of the experimental group drank approximately $0.190 \mathrm{ml} \mathrm{ED/g}$ of body weight/day. ED used in the study contained $32 \mathrm{mg}$ per $100 \mathrm{ml}$ caffeine, also water, sugar, citric acid, sodium citrate, taurine $(0.4 \%)$, inositol $(0.02 \%)$, niacin (7.92 mg per $100 \mathrm{ml})$, pantothenic acid (1.98 mg per $100 \mathrm{ml})$, B6 vitamin ( $2 \mathrm{mg}$ per $100 \mathrm{ml}$ ), sodium benzoate and potassium sorbate. The mean calorific energy value of $100 \mathrm{ml}$ was $185.5 \mathrm{~kJ}$ (43.7 kcal). Mean daily caffeine consumption was $20.77 \mathrm{mg}$ per rat. Both groups had an equal, unlimited access to food. After 8 weeks, the animals of both groups were decapitated and their hearts were collected for histological analysis. The study protocol was accepted by the Local Ethical Committee of the Medical University of Lublin (6a/2013).

\section{Histological procedures}

The collected material was fixed in $10 \%$ buffered formalin. Prepared organs were then embedded in paraffin blocks. Material was cut into $5 \mu \mathrm{m}$ thick slices and stained with haematoxylin and eosin (H\&E). Slices were analyzed using an Olympus BX41 light microscope with a digital camera and CellSens software.

\section{Immunohistochemical stainings}

Immunohistochemical stainings were done using antibodies against caspase 3 (WHO000836M2; Sigma-Aldrich, 1:40 dilution) and p53 (P5813-.2ML; Sigma-Aldrich, 1:200 dilution). Antigens were exposed by 3 cycles, each lasting 5 minutes, of heating in a microwave oven $(800 \mathrm{~W})$ while the slices were buffered with citrate buffer $(\mathrm{pH}=6)$. Endogenous peroxidase activity was inhibited by $0.3 \%$ methanolic solution of perhydrol $\left(\mathrm{H}_{2} \mathrm{O}_{2}\right)$. Normal serum was used for blockage of non-specific binding sites. Incubation with the primary antibody was done at a dilution indicated by the manufacturer at $4^{\circ} \mathrm{C}$ overnight. Subsequently, Post Primary Block and Polymer were used, and for visualization of the reaction, diaminobenzidine and hematoxylin. Negative control was performed in the same manner without the use of primary antibody. The analysis of the material was performed under light microscope $(20 \times$ and $40 \times)$. Cells with positive expression were determined as a percentage of total by counting 100 cells in 10 randomly chosen microscope fields under magnification. The intensity of caspase 3 and p53 protein expression was examined by way of CellSens software. Statistical analysis was performed with STATISTICA 12 software. U Mann-Whitney test was used for calculation of the statistical significance with the level of significance set at $\mathrm{p}<0.05$.

\section{RESULTS}

\section{H\&E staining}

Increased cardiomyocytes vacuolization was observed in the experimental (Fig. 1). In this group, the thickness of ventricle walls was $3.8 \pm 0.35 \mathrm{~mm}$, and in the control group, $3.62 \pm 0.25 \mathrm{~mm}$. The difference was statistically insignificant ( $p=0.6$, U Mann-Whitney Test). Moreover, the individual cardiomyocytes were slightly thicker in the experimental group $(15.37 \pm 4.10 \mu \mathrm{m})$ than in the control group $(13.83 \pm 4.71 \mu \mathrm{m})$, but the difference was also statistically insignificant $(\mathrm{p}=0.104692$, U Mann-Whitney Test).

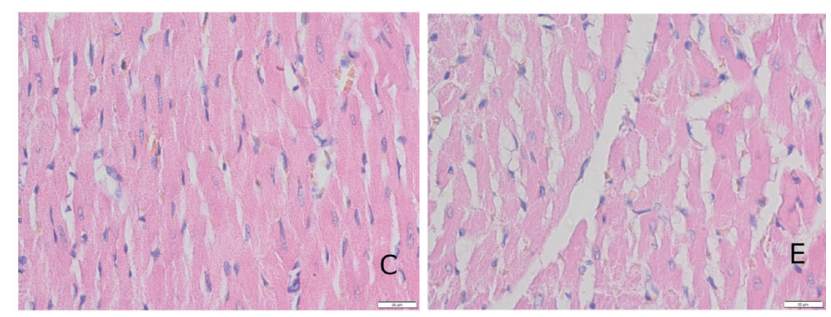

Figure 1. The microscopic view of the fragment of rat's heart ventricular wall. C - the control group, drinking water, E the experimental group drinking energy drinks. Increased vacuolization of cardiomyocytes in the experimental group is visible. H\&E staining. Magn. ca $400 \times$ 


\section{Caspase 3}

The analysis of the caspase 3 expression in the material revealed a higher frequency of caspase-positive cells in the experimental group $(50.75 \pm 13.17 \%)$ than in the control group $(15.42 \pm 4.94 \%)$. The difference was statistically significant ( $\mathrm{p}=0.000003$, U Mann-Whitney Test). In addition, the caspase 3 reaction revealed greater staining intensity in the cardiomyocytes of the experimental group, in comparison to the control group (Fig. 2).

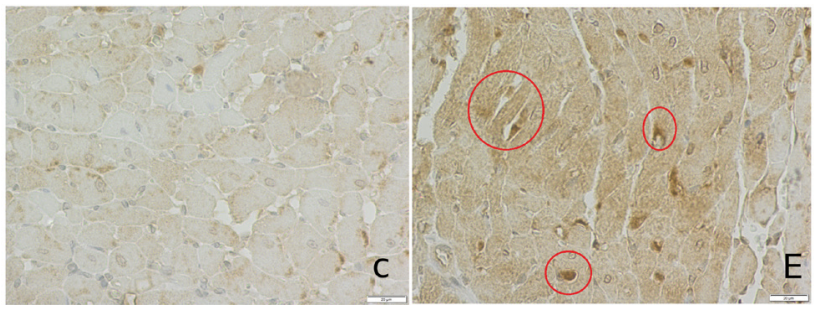

Figure 2. e microscopic view of e fragment of ras heart ventricular wall. C - the control group, E - the experimental group. A Higher expression of caspase 3 was seen in the cardiomyocytes of the experimental group. Increased caspase- 3 expression is marked by circles. Standard immunohistochemical staining with anticaspase-3 antibodies. Magn. ca 400x

\section{p53 protein}

The intensity of $\mathrm{p} 53$ expression was comparable in both the control and the experimental groups (Fig. 3). However, a difference in the location of immunoprecipitates was observed: in the control group, these were mainly located in cytoplasm $(85.68 \pm 9.40 \%)$ and to a lesser extent, in the nuclei $(40.63 \% \pm 12.80 \%)$; In the experimental group, however, immunoprecipitates were located more frequently in the nuclei $(78.95 \% \pm 13.20 \%)$ than in the cytoplasm $(41.5 \% \pm 11.09 \%)$. The differences in the localization of immunoprecypitates between the groups were statistically significant $(\mathrm{p}=0.012186$ (nuclei location) and $\mathrm{p}=0.030384$ (cytoplasm location) in the U Mann-Whitney Test ) (Fig. 4).

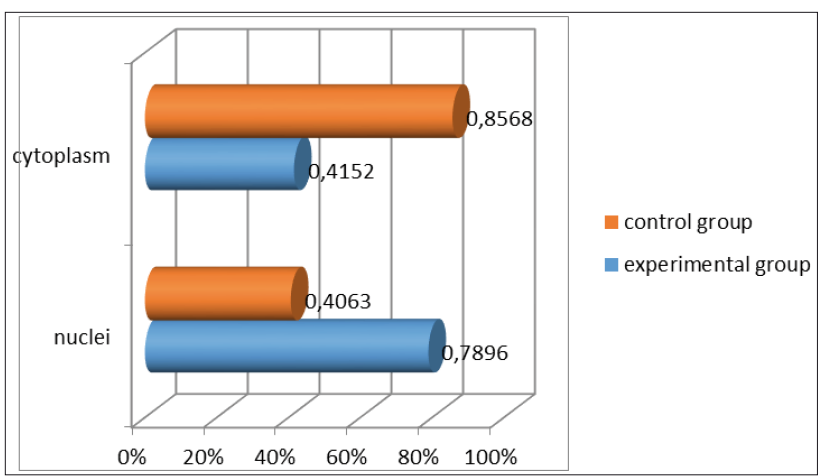

Figure 3. Percentage of positive reaction for p53 in the nuclei and cytoplasm of cardiomyocytesiof botn the experimental and control groups. Immunoprecipitations in the nuclei were observed more frequently in the experimental group than in the control group. The differences in the localization of immunoprecypitates between the groups were statistically significant $(\mathrm{p}=0.012186$ (nuclei location) and $\mathrm{p}=0.030384$ (cytoplasm location) in the U Mann-Whitney Test)

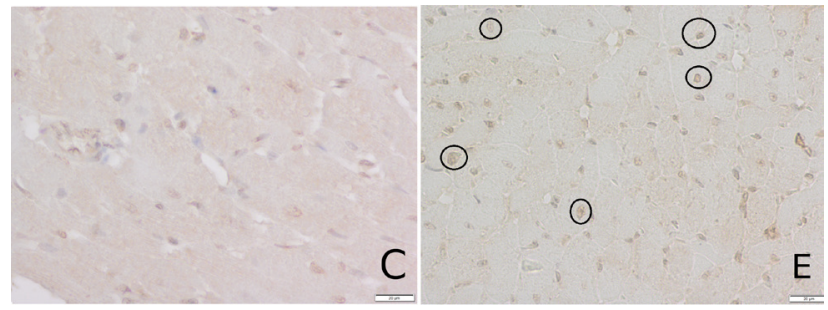

Figure 4. Microscopic view - immunohistochemical staining of rat heart fragments by way th the use of an antibody against prt, C - the control grop, - in the control group, we noted that the immunoprecypitates wer localized more frequently in the cytoplasm, rarely in the nuclei, E - the experimental grop, - herein, immunoprecypitates were localized more frequently in the nuclei (visible in the examination circles), rarely in the cytoplasm

\section{DISCUSSION}

Energy drinks purportedly improve concentration, lower fatigue and increase physical performance. They contain caffeine, taurine, sugar, vitamins, amino acids and herbal extracts [1]. Caffeine concentration varies between 32 and $100 \mathrm{mg}$ per $100 \mathrm{ml}$.

There are no legal restrictions in buying and selling energy drinks, therefore, they are freely available in almost every shop and gas station. Extensive advertising, the colourful packaging and noted short-term improvement after ED consumption has result in the high popularity of EDs. They are most popular among sportsmen, sports-wannabes, drivers and young people (including children) $[3,18]$.

Available literature points to risks related to youth ED consumption, especially when they are consumed together with alcohol [19]. ED consumption is linked to increased risk of various circulatory diseases such as arrhythmia, myocardial infarction, QT prolongation, aortic dissection and death due to cardiovascular reasons [20].

In this article, we focused on changes within cardiac muscle tissue and cardiomyocytes. Such changes could potentially be linked to the prior-mentioned clinical symptoms observed in ED consumers. According to available literature, not much has been published on tissue changes after ED consumption, more data is available on the relation between caffeine consumption and cardiovascular health. Caffeine may have positive and negative impacts upon the heart - it can both lower risks of cardiovascular diseases [21], but also due to increase in heart rate, it may lead to cardiac muscle ischaemia [22].

Taurine, another ED component, also affects the heart. As shown by previous studies, taurine acts condescendingly on the myocardium, mainly protecting against apoptosis [23-25]. Schaffer et al. indicate that taurine can reduce the impact of caffeine on the cardiovascular system [26]. The combination of these two substances, however, increase the strength of myocardial contractility after Ed ingestion [27]. Giles et al. examined the impact of these two substances on concentration and memory and revealed that they can act in different ways on the tissues and cells of various organs [28].

No definite conclusion can be made whether observed changes are caused by single ED ingredients or by synergistic action of more than one. Hence, it is far more possible 
that the real cause of the observed anomalies lies in the whole composition of ED.

In physiological conditions, apoptosis occurs in the heart at a low level and is related to normal tissue regeneration. An increased apoptotic rate can be, however, observed in various pathological states like ischaemia, hypoxia, increase $\mathrm{Ca}^{2+}$ levels and oxidative stress. Kunapuli S. et al. note that apoptosis can be increased in cardiac insufficiency [29].

In our analysis, we focused on the issue of apoptosis via rat cardiomyocytes as initiated by the consumption of ED. In doing so, we observed a significant increase in expression of caspase 3 in the cardiomyocytes of rats of the experimental group. As p53 was more frequently located in the nuclei, a pathological intracellular process may be suspected. The noted increased expression of caspase 3 in cardiomyocytes indicate that changes in the heart lead to cardiomyocytes apoptosis $[16,17]$. The intensified apoptosis caused by ED consumption, can lead to irreversible changes in the heart tissue, and to long-time consequences that are clinically observable. Finally, the seen decrease in cardiomyocytes number may lead to disturbances in the heart work and to homoeostasis of the body.

\section{CONCLUSIONS}

Energy drinks consumption leads to histopathological changes within the cardiomyocytes. Furthermore, the ED ingredients bring about increased cardiomyocytes apoptosis. This last could be the cause of cardiovascular disorders in the ED consumers

\section{ACKNOWLEDGEMENTS}

The authors declare that they have no conflict of interest.

\section{REFERENCES}

1. Bailey RL, Saldanha LG, Gahche JJ, Dwyer JT. Estimating caffeine intake from energy drinks and dietary supplements in the United States. Nutr Rev. 2014 Oct;72:9-13.

2. Lara B, Gonzalez-Millán C, Salinero JJ, Abian-Vicen J, Areces F, Barbero-Alvarez JC, et al. Caffeine-containing energy drink improves physical performance in female soccer players. Amino Acids. 2014 May;46(5):1385-92.

3. Usman A, Bhombal ST, Jawaid A, Zaki S. Energy drinks consumption practices among medical students of a Private sector University of Karachi, Pakistan. JPMA J Pak Med Assoc. 2015 Sep;65(9):1005-7.

4. Seifert et al. Health Effects of Energy Drinks on Children, Adolescents, and Young Adults. Pediatrics. 2011;127(3):511-528. PEDIATRICS. 2016 May 1;137(5):e20160454-e20160454.

5. Brothers RM, Christmas KM, Patik JC, Bhella PS. Heart rate, blood pressure and repolarization effects of an energy drink as compared to coffee. Clin Physiol Funct Imaging. 2016 Feb;n/a-n/a.

6. Grasser EK, Yepuri G, Dulloo AG, Montani J-P. Cardio- and cerebrovascular responses to the energy drink Red Bull in young adults: a randomized cross-over study. Eur J Nutr. 2014 Oct;53(7):1561-71.

7. Ilie G, Boak A, Mann RE, Adlaf EM, Hamilton H, Asbridge M, et al. Energy Drinks, Alcohol, Sports and Traumatic Brain Injuries among Adolescents. de Castro F, editor. PLOS ONE. 2015 Sep 16;10(9):e0135860.

8. Rutledge PC, Bestrashniy JRBM, Nelson TF. Problematic Drinking Among Postgraduate Students: Binge Drinking, Prepartying, and Mixing Alcohol With Energy Drinks. Subst Use Misuse. 2016 Jul 2;51(8):972-82.
9. Jonjev ZS, Bala G. High-energy drinks may provoke aortic dissection. Coll Antropol. 2013 May;37 Suppl 2:227-9.

10. Wiesmann UN, DiDonato S, Herschkowitz NN. Effect of chloroquine on cultured fibroblasts: release of lysosomal hydrolases and inhibition of their uptake. Biochem Biophys Res Commun. 1975 Oct 27;66(4):1338-43.

11. Avcı S, Sarıkaya R, Büyükcam F. Death of a young man after overuse of energy drink. Am J Emerg Med. 2013 Nov;31(11):1624.e3-1624.e4.

12. Nachmias B, Ashhab Y, Benyehuda D. The inhibitor of apoptosis protein family (IAPs): an emerging therapeutic target in cancer. Semin Cancer Biol. 2004 Aug;14(4):231-43.

13. Timmer JC, Salvesen GS. Caspase substrates. Cell Death Differ. 2007 Jan;14(1):66-72.

14. Walsh JG, Cullen SP, Sheridan C, Luthi AU, Gerner C, Martin SJ. Executioner caspase- 3 and caspase-7 are functionally distinct proteases. Proc Natl Acad Sci. 2008 Sep 2;105(35):12815-9.

15. Sznarkowska A, Olszewski R, Zawacka-Pankau J. [Pharmacological activation of tumor suppressor, wild-type p53 as a promising strategy to fight cancer]. Postepy Hig Med Doswiadczalnej Online. 2010 Aug 20;64:396-407.

16. Sznarkowska A, Olszewski R, Zawacka-Pankau J. [Pharmacological activation of tumor suppressor, wild-type p53 as a promising strategy to fight cancer]. Postepy Hig Med Doswiadczalnej Online. 2010 Aug 20;64:396-407.

17. Laptenko $\mathrm{O}$, Prives $\mathrm{C}$. Transcriptional regulation by p53: one protein, many possibilities. Cell Death Differ. 2006 Jun;13(6):951-61.

18. Seifert SM, Schaechter JL, Hershorin ER, Lipshultz SE. Health Effects of Energy Drinks on Children, Adolescents, and Young Adults. PEDIATRICS. 2011 Mar 1;127(3):511-28.

19. Marmorstein NR. Interactions Between Energy Drink Consumption and Sleep Problems: Associations with Alcohol Use Among Young Adolescents. J Caffeine Res. 2017 Sep;7(3):111-6.

20. Mangi MA, Rehman H, Rafique M, Illovsky M. Energy Drinks and the Risk of Cardiovascular Disease: A Review of Current Literature. Cureus [Internet]. 2017 Jun 7 [cited 2017 Oct 9]; Available from: http://www.cureus.com/articles/7289-energy-drinks-and-the-riskof-cardiovascular-disease-a-review-of-current-literature

21. You D-C, Kim Y-S, Ha A-W, Lee Y-N, Kim S-M, Kim C-H, et al. Possible Health Effects of Caffeinated Coffee Consumption on Alzheimer's Disease and Cardiovascular Disease. Toxicol Res. 2011 Mar 1;27(1):7-10.

22. Berger AJ, Alford K. Cardiac arrest in a young man following excess consumption of caffeinated "energy drinks." Med J Aust. 2009 Jan 5;190(1):41-3.

23. Xu Y, Saini H, Zhang M, Elimban V, Dhalla N. MAPK activation and apoptotic alterations in hearts subjected to calcium paradox are attenuated by taurine. Cardiovasc Res. 2006 Oct 1;72(1):163-74.

24. Oriyanhan W, Yamazaki K, Miwa S, Takaba K, Ikeda T, Komeda M. Taurine prevents myocardial ischemia/reperfusion-induced oxidative stress and apoptosis in prolonged hypothermic rat heart preservation. Heart Vessels. 2005 Nov 25;20(6):278-85.

25. Takatani T, Takahashi K, Uozumi Y, Matsuda T, Ito T, Schaffer SW, et al. Taurine prevents the ischemia-induced apoptosis in cultured neonatal rat cardiomyocytes through Akt/caspase- 9 pathway. Biochem Biophys Res Commun. 2004 Apr;316(2):484-9.

26. Schaffer SW, Shimada K, Jong CJ, Ito T, Azuma J, Takahashi K. Effect of taurine and potential interactions with caffeine on cardiovascular function. Amino Acids. 2014 May;46(5):1147-57.

27. Doerner JM, Kuetting DL, Luetkens JA, Naehle CP, Dabir D, Homsi $\mathrm{R}$, et al. Caffeine and taurine containing energy drink increases left ventricular contractility in healthy volunteers. Int J Cardiovasc Imaging. 2015 Mar;31(3):595-601.

28. Giles GE, Mahoney CR, Brunyé TT, Gardony AL, Taylor HA, Kanarek RB. Differential cognitive effects of energy drink ingredients: Caffeine, taurine, and glucose. Pharmacol Biochem Behav. 2012 Oct;102(4):569-77.

29. Kunapuli S, Rosanio S, Schwarz ER. "How Do Cardiomyocytes Die?” Apoptosis and Autophagic Cell Death in Cardiac Myocytes. J Card Fail. 2006 Jun;12(5):381-91. 